\title{
The nature and incidence of injuries in morris dancers
}

\author{
A.R. Tuffery, PhD \\ Department of Physiology, Trinity College, Dublin, Ireland
}

\begin{abstract}
Morris dancing includes several distinct forms of vigorous traditional dance with about 5000 amateur participants in the UK and Ireland. Questionnaires were sent to over 500 registered morris sides. The response rate was 29 per cent with 129 acute injuries and 47 chronic injuries including one fatality. The commonest acute injuries were to ankle (33 per cent) and calf ( 22 per cent). The back was the commonest site of chronic injury, with a high contribution from occupational and other sources. Morris dancing is a relatively low-risk activity, but injuries could be prevented by improving the fitness of dancers and selecting safer surfaces to dance on. The severity of injuries could probably be reduced by seeking more and better treatment.
\end{abstract}

Keywords: Survey, dancing, injuries

\section{Introduction}

Injuries incurred during sports and recreation represent a significant public health problem, and while much information is available about the major organised sports, there is little information about activities which are more loosely organised or have lower injury or participation rates ${ }^{1}$. A survey of the nature and incidence of injuries occurring in relation to morris dancing is of interest because there are very few such studies of injuries related to dancing of any type $^{2,3}$, and all refer specifically to professional or student dancers. Morris dancing is performed by amateurs and encompasses several distinct types of vigorous traditional dance which will not be distinguished in this report.

\section{Method}

Late in 1987 a detailed questionnaire was sent to each of the 523 morris dance groups ('sides') registered with The Morris Federation, Open Morris and The Morris Ring, which represent morris dancers in the UK and Ireland. A copy of the questionnaire is available from the author. The coded data in the form of a database or spreadsheet is also available (with a key) on receipt of a Macintosh ${ }^{\mathrm{TM}}$ disc. The aims of the survey were to find the incidence and nature of acute and chronic injuries occurring in morris dancers in the calendar year

Address for correspondence: Dr A.R. Tuffery, Lecturer, Department of Physiology, Trinity College, Dublin 2, Ireland

(C) 1989 Butterworth \& Co (Publishers) Ltd

0306-3674/89/030155-06\$03.00
1987 and to attempt to determine the contributory causes of those injuries.

The protocol of the survey meets most of the criteria recommended by the largest and most effective studies $^{4}$ except that this study was retrospective. As Bowling has pointed out, this may mean that some injuries may be missed because of the dancers' retirement ${ }^{3}$.

The questionnaire sought information concerning the make-up and activity of the side, acute injuries and chronic injuries. For each injury, specific questions were asked about the treatment and severity of the injury and possible contributory factors. Respondents were encouraged to comment freely.

Acute injuries were defined as those caused by a specific event during dancing in practice or performance and which required medical attention or caused considerable pain during dancing. A chronic injury was defined as an injury aggravated by morris dancing and which either prevented dancing or caused considerable discomfort. The severity of injuries was graded according to the criteria of the US National Athletic Injury/Illness Reporting System ${ }^{4}$. A minor injury restricted the dancer's participation for up to seven days, a moderate injury for 8-21 days and a major injury for more than 21 days. The last two categories are grouped as significant injuries. Severe injuries include those having such consequences as death, major paralysis and amputation. Treatment was defined to exclude first aid (except where specifically mentioned) and rest alone.

The sample sizes may vary because of non-responders or because of individuals with more than one injury. The chi-squared test was used to test for significant differences in incidences. A probability of less than 0.05 was regarded as statistically significant. Percentages have been rounded to the nearest whole number.

\section{Results}

Of the 523 sides circulated, 149 (29 per cent) responded; 87 (58 per cent) reported 129 acute injuries and 47 chronic injuries, with one fatality due to a heart attack in a male aged over 60 .

It was estimated from the activity data and information about the sex composition of sides that females and males were equally represented in the sides reporting injuries. Females had 59 per cent of the acute 

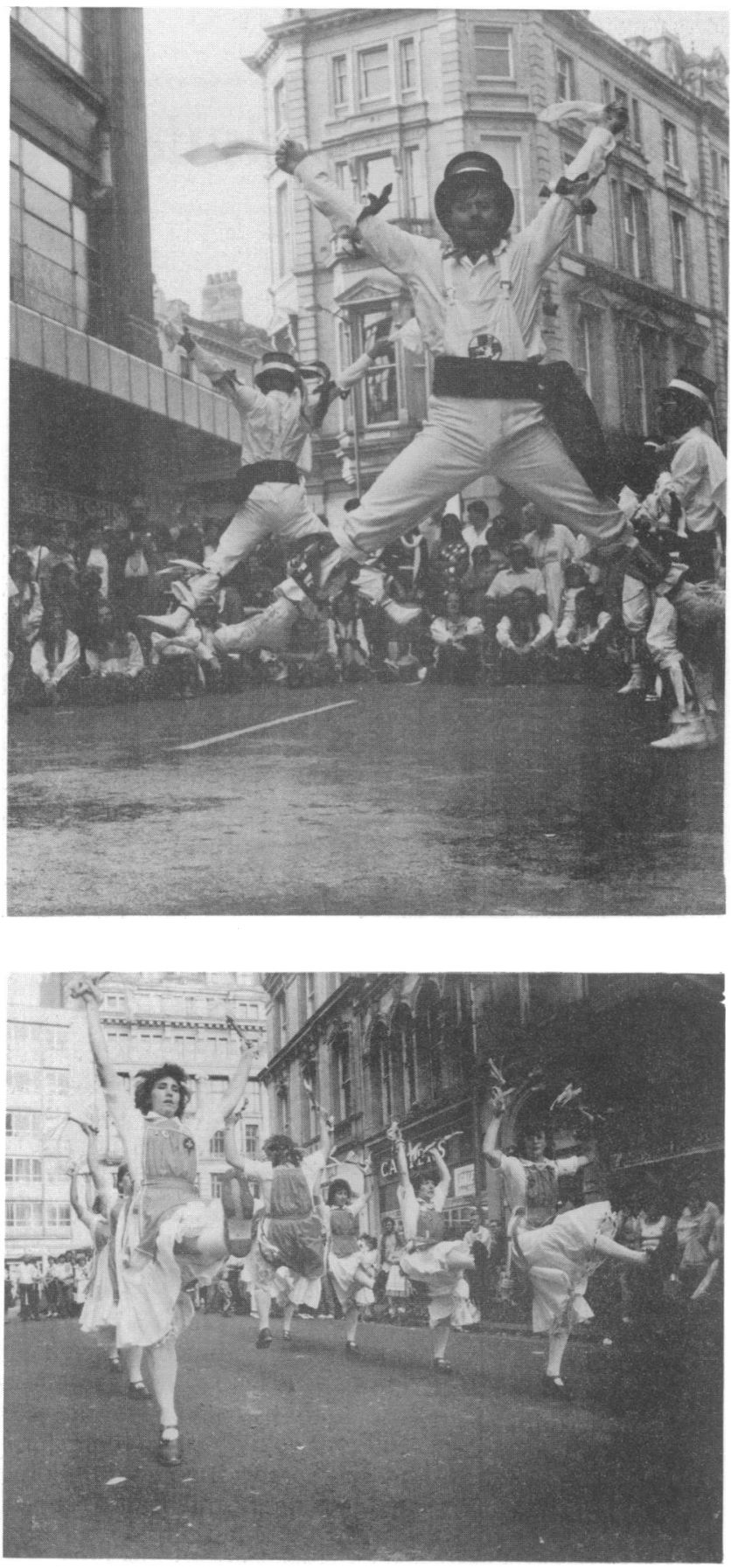

injuries. There were no significant differences between the age distributions, with $57-59$ per cent of injuries occurring in the 31-40 year-old age-group in each sex. Chronic injuries tended to occur in slightly older subjects than acute injuries $(P<0.001$ Table 1$)$.

\section{Activity}

Information about the number of dancers and the number of practices and performances was used to estimate the total activity of the respondent sides. The sides represented about 1337 dancers whose activity was evenly split between practice and performance. The estimated total activity was 135406 person hours or 79027 athlete-exposures, where athlete-exposures equals (number of dancers $\times$ number of practices) + (number of dancers $\times$ number of performances) ${ }^{4}$. The

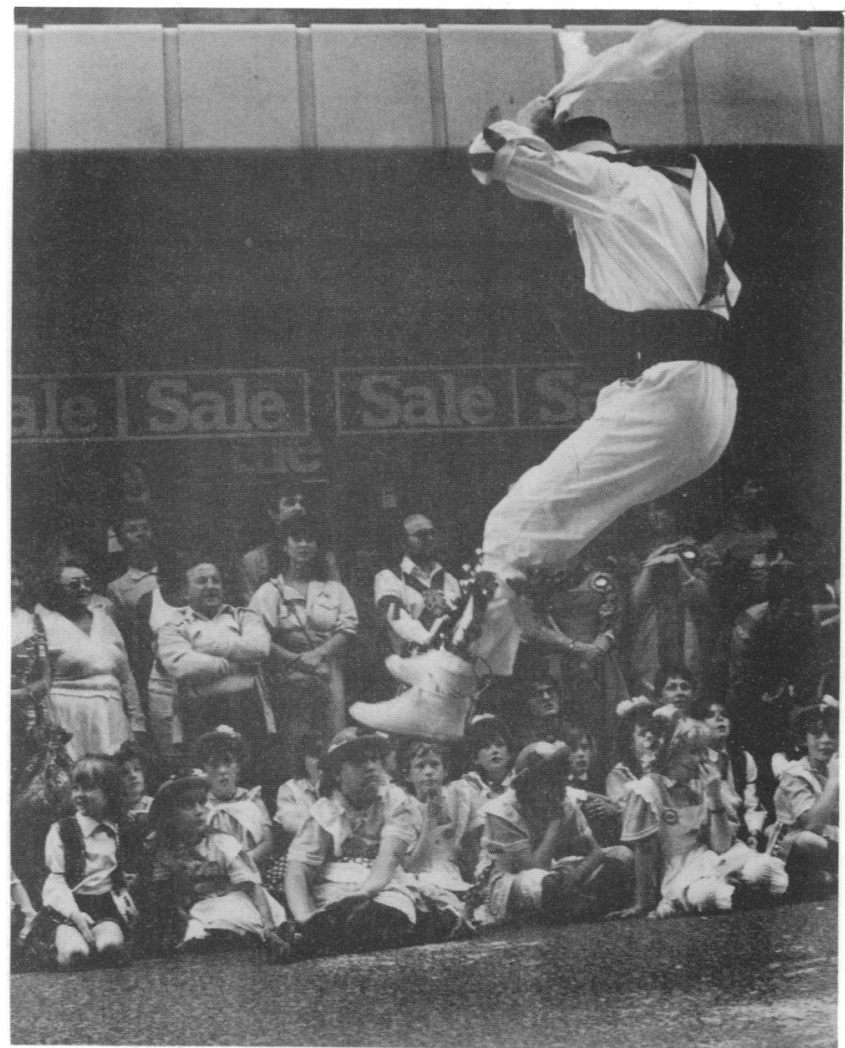

Figures 1-3. Action shots of Morris dancing showing high degree of exertion

overall incidence of acute injuries is 9.5 per cent or approximately one injury per 1000 participant hours of morris dancing or 1.5 per 1000 athlete-exposures.

\section{Acute injuries}

Injuries to the lower limb accounted for 70 per cent of the total, with injuries to calf and ankle comprising over half of the acute injuries reported (Table 2). Other significant sites were knee, finger and foot (including toes). Only 26 per cent of those with acute injuries had warmed up before dancing. The surface was considered to have contributed to the injury in 39 per cent of cases.

Table 1. The percentage age and sex distribution of the respondents with acute injuries compared with the age distribution of those with chronic injuries

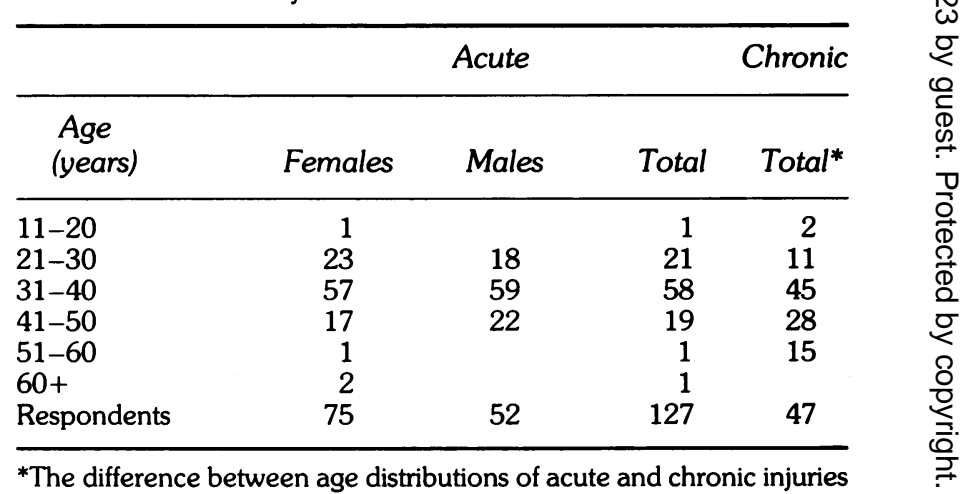

*The difference between age distributions of acute and chronic injuries is statistically significant $(P<0.001)$ 
Table 2. The site of acute injuries in females (77 injuries) and males (52 injuries)

\begin{tabular}{lrrrrrr}
\hline & \multicolumn{2}{c}{ Females } & \multicolumn{2}{c}{ Males } & \multicolumn{2}{c}{ Total } \\
& $\mathrm{n}$ & $(\%)$ & $\mathrm{n}$ & $(\%)$ & $\mathrm{n}$ & $(\%)$ \\
\hline Ankle & 26 & $(34)$ & 16 & $(31)$ & 42 & $(33)$ \\
Calf & 20 & $(26)$ & 8 & $(15)$ & 28 & $(22)$ \\
Finger* & 3 & $(4)$ & 7 & $(13)$ & 10 & $(8)$ \\
Knee* & 3 & $(4)$ & 7 & $(13)$ & 10 & $(8)$ \\
Foot/toe & 6 & $(8)$ & 5 & $(10)$ & 11 & $(9)$ \\
Othert & 19 & $(25)$ & 9 & $(17)$ & 28 & $(22)$ \\
\hline
\end{tabular}

*Difference between males and females $P<0.05$

tIncludes all sites with fewer than 4 cases

There was no difference between males and females in the percentage frequency of ankle injuries, but males had significantly higher incidences of injuries to fingers and knee, and females had a higher incidence of calf injuries, although the last was not statistically significant. A brief consideration of the injuries to these principal sites follows.

Finger Eight of the ten finger injuries were caused by sticks used during the dance; five in practice and five in performance. Bruising was the commonest type of injury (four cases). There were three cuts. All finger injuries occurred in the middle or late in practice or performance. Five had treatment other than first aid, four as hospital outpatients. Three injuries were of moderate severity. The anecdotal evidence suggested that males tended to have injuries more serious than simple bruising and tended to be off dancing for longer.

Knee Seven of the ten knee injuries were in males. There was a large diversity of types of injury (only sprain occurs more than once). Five cases were medically diagnosed. Six knee injuries occurred in performance and four in practice. The dancing surface was implicated in only two cases. Only two injuries occurred early in dance and one after warm-up. Six of the injuries were major, that is, they prevented dancing for more than 21 days. Three caused cessation of dancing for more than two months.

Calf There were 28 calf injuries ( 20 in females, 8 in males). All except one was described as a muscle injury. About half were medically diagnosed. The majority of calf injuries occurred in performance (68 per cent in females, 88 per cent in males). All seven injuries occurring in practice were not later than halfway through and only one person had warmed up. Of the 20 injuries occurring in performance, only five were late in the day. The dancing surface was implicated in only five cases. Two of these were cases of multiple injuries, including ankle injuries. Ten of the cases had no treatment; six were hospital in-patients. Only seven had later treatment, including physiotherapy. Sixteen of the injuries were moderate and 12 major, with one preventing participation for up to six months. Two specific movements accounted for over half (54 per cent) of the total calf injuries. One involved striking the ground with an almost vertical movement (rant/polka step) while the other involved landing on one leg (caper).
Table 3. The severity of acute and chronic injuries classified according to the restriction of participation*

\begin{tabular}{lcccc}
\hline & \multicolumn{2}{c}{ Acute } & \multicolumn{2}{c}{ Chronict } \\
& $\mathrm{n}$ & $(\%)$ & $\mathrm{n}$ & $(\%)$ \\
\hline Minor & 30 & $(24)$ & 4 & $(10)$ \\
Moderate & 42 & $(33)$ & 12 & $(30)$ \\
Major & 54 & $(43)$ & 24 & $(60)$ \\
\hline
\end{tabular}

*Minor $<7 \mathrm{~d}$

Moderate 8-21d

Major $>21 d^{4}$

tDifference between acute and chronic injuries is statistically significant $(P<0.05)$

Ankle There were 42 ankle injuries. Almost all (39) were recorded as sprains and 34 were diagnosed medically. Only nine received initial treatment other than rest or first aid. Seventeen received further treatment and six received physiotherapy. Most ankle injuries (70 per cent) were major as defined by the time that dancers were unable to participate, with 12 (30 per cent) preventing participation for over three months. Ankle injuries occurred equally at all times in the dancing session, but only in one case within minutes of starting dancing. The surface was implicated in 25 cases (61 per cent). In sixteen cases ( 38 per cent) clogs were worn. Of these, 13 (81 per cent) considered that the surface was implicated. This was significantly higher $(P<0.05)$ than in dancers not wearing clogs.

Only 24 per cent of all acute injuries were minor; 33 per cent were moderate and 43 per cent major (Table 3 ). There was a fairly obvious relationship between the site of injury and the time taken to return to dancing. Thus, finger injuries did not greatly restrict participation, but ankle, knee and calf injuries did.

About one third ( 34 per cent) of injuries received no treatment other than rest immediately after injury. Another third ( 39 per cent) were treated with first aid only. The remaining 27 per cent attended hospital casualty departments or general practitioners. Over half (56 per cent) of the injuries received no subsequent treatment. Approximately similar numbers were treated as hospital outpatients, by general practitioners and by physiotherapists (15 per cent, 13 per cent and 11 per cent, respectively). Only one injury was treated at a specialist sports injuries clinic.

It is difficult to summarize the different types of movement which were recorded as taking place at the time of injury (106 cases) because of the necessity for technical terms. However, the commonest movements were rant or polka steps (vigorous stepping with emphasis on the striking of the ground) which were implicated in 22 per cent of acute injuries; capers (a high leap landing on one leg) - 10 per cent; turns 10 per cent. The use of sticks accounted for a further 10 per cent, mainly the finger injuries as noted earlier.

\section{Chronic injuries}

The total number of chronic injuries reported was 47 . Only five (11 per cent) were in females; three knee injuries and two shin injuries. This is significantly $(P<0.001)$ less than the 59 per cent of acute injuries in females. Over two-thirds (68 per cent) of chronic injuries were diagnosed medically, compared with 55 
Table 4. The site of chronic injuries in females (5 injuries) and males (42 injuries)

\begin{tabular}{lccrrrr}
\hline & \multicolumn{2}{c}{ Females } & \multicolumn{2}{c}{ Males* } & \multicolumn{2}{c}{ Total } \\
& $\mathrm{n}$ & $(\%)$ & $\mathrm{n}$ & $(\%)$ & $\mathrm{n}$ & $(\%)$ \\
\hline Knee & 3 & $(6)$ & 19 & $(40)$ & 22 & $(47)$ \\
Back & & & 6 & $(13)$ & 6 & $(13)$ \\
Ankle & & & 5 & $(11)$ & 5 & $(11)$ \\
Calf & & & 3 & $(6)$ & 3 & $(6)$ \\
Shin & 2 & $(4)$ & 1 & $(2)$ & 3 & $(6)$ \\
Foot/toe & & & 3 & $(6)$ & 3 & $(6)$ \\
Othert & & & 5 & $(11)$ & 5 & $(11)$ \\
\hline
\end{tabular}

*Difference between overall incidences in males and females is statistically significant $(P<0.001)$

tIncludes all sites with only one case

per cent of acute injuries (not significantly different). Only 26 per cent normally warmed up before dancing, the same proportion as in acute injuries.

Injuries to knee and back accounted for 60 per cent of all chronic injuries, with knee injuries making up 47 per cent. This is significantly greater $(P<0.001)$ than the incidences of acute injuries to these sites.

Half of the 22 knee injuries and half of the six back injuries were associated with movements which may be described as weight-bearing. The rant/polka step accounted for almost a third of chronic knee injuries. Bending and galleys (a movement requiring rotation of the lower leg with a fixed, raised thigh) accounted for the other back injuries. The back injuries were regarded as serious as indicated by the fact that four received subsequent treatment.

Injuries incurred in activities other than morris dancing were implicated as contributing to the chronic injuries in 19 (42 per cent) cases. Eight, including four knee injuries, were related to other sports, and five (four back injuries and one multiple injury) cited lifting, usually at work.

The majority ( 57 per cent) occurred in the year of the survey or the preceding year with small numbers in each of the previous eight years. One dancer had had a chronic ankle injury for 24 years. One third of the knee injuries occurred in the year preceding the survey.

There was no significant difference between the proportions of acute and chronic injuries receiving no treatment other than rest ( 46 per cent and 33 per cent, respectively). Three chronic injuries (seven per cent) were treated as hospital in-patients, 24 per cent received physiotherapy and five per cent saw either a chiropractor or an osteopath. Those with acute injuries had no significant numbers who received any of these categories of initial treatment, but 12 per cent subsequently had physiotherapy and one per cent became hospital in-patients.

Of the 42 respondents, 86 per cent were prevented from dancing occasionally, 12 per cent most of the time, and only one individual was continuously unable to dance. Six cases involved pain which occurred after dancing and several respondents mentioned that this was not sufficient to deter them from continuing to dance. According to the time that dancers were unable to participate, 10 per cent of chronic injuries were classified as minor, 30 per cent as moderate and 60 per cent as major. This was not significantly different from the pattern in acute injuries.

\section{Discussion}

This discussion will compare the results of this study with other relevant studies and will attempt to draw conclusions and make suggestions for reducing the occurrence of injuries.

\section{Incidence of injuries}

In this survey there was an overall incidence of 9.1 per cent (i.e. 9.1 acute injuries per 100 dancers). There are no previous studies of morris dancers, so it is not possible to make direct comparisons, but comparisons with other activities may be helpful. In general the studies which have been selected have survey data which can be interpreted quantitatively. Studies where the sample is taken from those presenting for medical treatment have been excluded on the grounds that they represent a self-selected and therefore biased estimate of incidence. The object was to give an impression of the relative risk of morris dancing.

The incidence of sports-related injuries in adults in Greater Helsinki (population 600000,75 per cent of whom take physical exercise) was 6.7 per cent in one year ${ }^{5}$. In a sample of 726 aerobic dancers, 49 per cent reported dance-related injuries ${ }^{6}$. However, this study was not restricted to a particular time-period so it is difficult to evaluate this figure which may be an aggregate over several years. The mean level of activity was 195 minutes per week, which is probably not greatly higher than that of many morris dancers. A survey of non-contact sports in about 11000 US high-school children gave an incidence of about 25 per cent ${ }^{7}$. According to Vanderfield who collated six studies published between 1971 and 1982, rugby football has an average injury rate of 9.3 injuries per 1000 'player/ games' (roughly equivalent to athlete-exposures; range 30.5-197.7) ${ }^{8}$. American football has an incidence of injuries of over 90 per cent or 11 per 1000 athlete-exposures ${ }^{4}$. The corresponding figure from our study is 1.5 per 1000 athlete-exposures.

Taking all these studies together, it is clear that morris dancing is a relatively low-risk activity, only slightly higher than the overall figure for the population as a whole, and considerably lower than both non-contact sports and sports with a high level of physical contact.

\section{Site of injury}

There was a clear distinction between acute and chronic injuries. In the former, ankle and calf injuries were the most common. Knee injuries were the commonest chronic injuries. For comparison, data from studies of dance students ${ }^{2}$, professional dancers ${ }^{3}$, and aerobic dancers ${ }^{6}$ are given in Table 5 , together with the acute and chronic injuries from this study. None of these other studies distinguished between acute and chronic injuries. The values are rounded to the nearest whole number.

The differences between the data on morris dancers and dance students are perhaps more striking than the similarities. This could be explained by the fact that dance students practice for up to six hours a day, six days a week. Consequently their injuries are much more likely to be due to long-term stress, rather than the acute twists, tears and sprains common among the morris injuries. This view is supported by the fact that 
Table 5. Percentage of injuries in specific sites in aerobic dancers $^{6}$, dance students ${ }^{2}$, professional dancers ${ }^{3}$ and in morris dancers

\begin{tabular}{lrrrrr}
\hline & & & & \multicolumn{2}{c}{ Morris Survey } \\
& Aerobics & Students & Dancers & Acute & Chronic \\
\hline Back & 13 & 10 & 26 & 2 & 13 \\
Knee & 9 & 18 & 12 & 6 & 47 \\
Shin & 25 & 11 & - & 1 & 6 \\
Ankle & 12 & 18 & 19 & 32 & 11 \\
Foot & 6 & 9 & 16 & 7 & 2 \\
Toe & - & 6 & & 2 & 4 \\
Calf & 6 & - & - & 21 & 0 \\
\hline
\end{tabular}

- indicates no data

Ankle includes Achilles tendon in students

Foot includes toes in dancers

the closest comparisons are between the chronic injuries and the dance students, especially in the back, ankle and toe. The much higher incidence of chronic knee injuries in the morris dancers might be explained by their continuing to dance with an injury which would exclude dance students from further participation. The incidence of acute foot injuries in morris dancers is quite similar to that of the students. Unfortunately, no information is available about their nature in the dance students.

The data on injuries in professional dancers (classical ballet and modern dance) refers only to injuries in the six months preceding the survey ${ }^{3}$. Overall, these data more closely resemble the aerobic dancers and the dance students more than the morris dancers, although the incidence of back and foot injuries is much greater. Unfortunately, the results for the thigh and leg were pooled in this study, so no comparison can be made for the calf injuries.

The data on aerobic dancers ${ }^{6}$ are similar to those on morris dancers in relation to foot, including toe, and knee injuries, but more closely resemble the dance students in the incidence of back and ankle injuries.

It appears from these comparisons that the pattern of injuries found in morris dancers is quite distinctive with the high incidence of ankle injuries as the most striking feature. This may be related to the fact that morris dancers dance on a variety of surfaces, some of which are unsuitable, whereas professional dancers usually dance on specially prepared surfaces.

\section{Possible causes of injuries}

Knee and calf injuries which form a significant proportion of the acute injuries share an almost total absence of any suggestion that the surface contributed to the injury. Knee injuries tended to occur late in the dancing session and this suggests that relative unfitness is the root cause.

It is noteworthy that all the knee injuries were in individuals involved in a dance style where the emphasis is on achieving lift-off usually from one leg, followed by landing on one leg. In contrast to the knee injuries, the calf injuries tended to occur early in the dancing session, which may imply that failure to warm up as well as unfitness may be contributory.

Ankle injuries comprised about a third of all acute injuries and were serious in that 85 per cent were medically diagnosed and 69 per cent caused more than a month off dancing. Unlike knee and calf injuries, the dancing surface was implicated in over half (55 per cent) of the cases. It seems reasonable to conclude that the principal causes of ankle injuries were the selection of an inappropriate surface to dance on, and an insufficient level of fitness. Warming up may not be as important for ankle injuries as for the calf injuries.

The low incidence of shin injuries is perhaps surprising (only six per cent of chronic injuries), although Bowling made a similar observation ${ }^{3}$. It was expected that 'shin splints' or soreness would be a common complaint. However, it may be that, although 'shin splints' occur, they do not interfere with dancing, because most dancers would dance not more than twice a week. This is in contrast to dance students or other athletes whose higher level of activity would mean that relatively slight injuries would interfere with their practice or performance.

\section{Sex differences}

The apparently higher incidence of calf injuries in females was not statistically significant. The most striking sex differences in acute injuries are the higher incidences of finger and knee injuries in males (Table 2). The finger injuries, which are virtually all associated with the use of sticks, can perhaps be accounted for by the greater robustness or lesser precision of sticking in males.

Other studies have sometimes ${ }^{9}$ but not always ${ }^{7}$ found a higher incidence of injuries in females. The usual reason for this is that females tend to have a lower level of fitness initially, and the difference tends to disappear as a sport gets more organized and the training facilities for females improve. A second contributory factor may be the shortness of the calf muscles in females, which would be exaggerated by the regular wearing of shoes with higher heels. If this is a factor, it would emphasise the need for females especially to undertake regular stretching exercises.

Females had only 11 per cent of the chronic injuries. This may reflect the pattern of acute injuries, since almost half of the chronic injuries reported were knee injuries which in turn occur more commonly in males.

\section{Treatment of injuries}

Most acute injuries did not receive initial treatment other than rest or first aid. Only about half of the injuries did have some subsequent treatment, in contrast to professional dancers who all sought treatment ${ }^{3}$. About two per cent were hospital in-patients, and 12-15 per cent were outpatients who saw a general practitioner or a physiotherapist. The incidence of physiotherapy seems low in relation to the number of ankle, knee and calf injuries, which might be expected to benefit from it. Indeed, current medical opinion is that a complete recovery is expected when soft tissue injuries are correctly treated ${ }^{10}$. The low rate of seeking specialist medical help may indicate that the physical demands of morris dancing are not taken sufficiently seriously by the dancers themselves. Other comparable amateur athletes may be much more willing to seek treatment.

The proportion of chronic injuries receiving treatment was 54 per cent, slightly higher than in acute injuries. The proportion receiving physiotherapy was 
24 per cent, double that for the acute injuries. This is not surprising because, by their nature, chronic injuries give persistent discomfort and sufferers are therefore more likely to seek treatment.

As might be expected, the time off dancing is greater for chronic injuries $(P<0.05)$. Because of the nature of the survey the time off may be an underestimate because, for example, a serious injury in June cannot cause more than six months off dancing since the survey was conducted in the following January. Also, the chronically injured may give up dancing altogether and therefore be lost to the survey. These considerations may also explain the very high incidence of chronic injuries in the two years immediately preceding the survey date.

Finally, it is possible to make some specific suggestions for the reduction of the incidence and severity of injuries incurred in morris dancing. An increased level of fitness, warming up and avoidance of uneven dancing surfaces would probably reduce the incidence of injuries. A greater provision of first aid (only 26 per cent of sides carry a first aid kit) and a greater willingness to seek professional treatment would reduce the severity of injuries and lead to a fuller recovery and possibly minimise the tendency for recurrence of injury ${ }^{1}$.

\section{Acknowledgements}

It is a pleasure to acknowledge the assistance of all the morris dancers who completed the questionnaire and the work undertaken by Sue Swift, Gordon Monks and Keith Francis of The Morris Federation, Open Morris and The Morris Ring as well as Roger Luckwill (Dublin City Morris Dancers), Stella New (Royal Manor Morris and Acorn Morris) and Roy Pinder (Gibbet Hill Morris). I am also grateful to Dr Mary Kelly and Professor Moira O'Brien of the departments of physiology and anatomy, respectively, Trinity College, Dublin for useful discussions. Photographs courtesy of D. Rowe.

\section{References}

1 Kraus, J.F. and Conroy, C. Mortality and morbidity from injuries in sports and recreation Annual Review of Public Health 1984, 5, 163-192

2 Shaw, J. 1977 'The Nature, Frequency and Patterns of Dance Injuries; a Survey of College Dance Students (M.A. thesis, University of Utah, Salt Lake City) Cited in Arnheim, D.D. Dance Injuries: Their Prevention and Cure, 2nd edn. London 1980, Mosby

3 Bowling, A. Injuries to dancers: prevalence, treatment and perception of causes $\mathrm{Br}$ Med J 1989, 298, 731-734

4 Powell, J.W. 'Pros and cons of data-gathering mechanisms' In Sports Injuries. The Unthwarted Epidemic, Ch. 3, Vinger, P.F. and Hoerner, E.F. eds. London 1982, John Wright PSG Inc

5 Sandelin, J., Santavirta, S., Lättilä, R., Vuolle, P. and Sarna, S. Sports injuries in a large urban population: occurrence and epidemiological aspects Int J Sports Med $1987,8,61-66$

6 Rothenberger, L.A., Chang, J.I. and Cable, T.A. Prevalence and types of injuries in aerobic dancers $A m \mathrm{~J}$ Sports Med 1988, 16, 403-407

7 Shively, R.A., Grana, W.A. and Ellis, D.E. High School sports injuries The Physician and Sportsmedicine $1981,9,46-50$

8 Vanderfield, G. 'Rugby' In Sports Injuries. Mechanism, Prevention, and Treatment, Ch. 2, eds Schneider, R.C., Kennedy, J.C. and Plant, M.L. London 1985, Williams \& Wilkins

9 Haycock, C.H. 1982 'Women in Sport' In Sports Injuries. The Unthwarted Epidemic Ch. 9, Vinger, P.F. and Hoerner, E.F. eds. London 1982, John Wright PSG Inc

10 Howse, J. and Hancock, S. 'Women in Sport' In Sports Injuries. The Unthwarted Epidemic Ch 9 Vinger, P.F. and Hoerner, E.F. eds. London 1982, John Wright PSG Inc 